\title{
Comparison of Electrogastrograms in Seated and Supine Positions Using Wayland Algorithm
}

\author{
Yasuyuki Matsuura ${ }^{1, *}$, Hiroki Takada² \\ ${ }^{1}$ Department of Cross Cultural Studies, Gifu City Women's College, 501-0192, Japan \\ ${ }^{2}$ Department of Human \& Artificial Intelligence Systems, Graduate School of Engineering, University of Fukui, 910-8507, Japan
}

\begin{tabular}{l} 
A R T I C L E I N F O \\
\hline Article history: \\
Received:18 December, 2018 \\
Accepted:26 June, 2019 \\
Online: 11 July, 2019
\end{tabular}

Keywords:

Electrogastrogram

Gastrointestinal motility

Postural Change

Wayland algorithm

\begin{abstract}
A B S T R A C T
In previous study, it is difficult to estimate the biological states such as postural ones and aetas in accordance with the previous analysis of electrogastrograms (EGGs), basically the frequency resolution of healthy individuals. In addition, few studies have compared gastric myoelectrical activity in supine and sitting posture. The aim of this study is to evaluate the severity of $3 D$ sickness in daily life. The authors herein conduct nonlinear analysis of EGGs of the healthy young and give the feasibility to discriminate the postural differences. It is suggested that the EGGs acquired in the seated position have increased wavelength irregularity and attractor complexity than those acquired in the supine position.
\end{abstract}

\section{Introduction}

There are a myriad of activities in our sitting posture, including paper work, light work and others, and fatigue accumulates if this position is maintained for long periods. People usually sleep and respite in a supine position. Likewise, sick people and those affected by 3D sickness take a break in the supine posture. Moreover, humans hardly get motion sickness while maintaining a supine position [1]. In contrast, a seated position can lead to experience both motion sickness, especially in moving ships, cars, and trains, and visually induced motion sickness (VIMS) that occurs while viewing stereoscopic movies. Still, few studies have made a comparison between gastrointestinal motilities in supine and those in seated posture to evaluate the severity of the VIMS.

One of the most relevant theories for VIMS is the sensory conflict theory [2-4]. According to this theory, sensory mismatch between visual and vestibular signals would be induced in virtual space compared to the real world [5]. Moreover, close anatomical and electrophysiological relationships between the autonomic and vestibular nervous systems have been reported [6]. It has been known that there is interaction between vestibular and autonomic nervous systems in the physiological fields; the motion sickness might affect both systems because dizziness and hidropoiesis are induced by the reaction of the autonomic nervous system due to the excessive stimulus for the vestibular system. For instance, it has been reported that the level of histamine increased in the

*Yasuyuki Matsuura, 7-1 Hitoichiba Kitamachi Gifu 501-0192 Japan, +81-58-

296-3131 \& matsuura@gifu-cwc.ac.jp

www.astesj.com

https://dx.doi.org/10.25046/aj040405 hypothalamus and brainstem in rats due to the rotation stimuli, which is possibly associated with vomiting during the motion sickness [7]. In addition, motion sickness seems to be induced by the contradiction among visual, vestibular, auditory, and proprioceptive inputs. These sensory informations can be compared with the previous experiences. The sensory informations are expected from the previous ones, however, they might be deviating from the previous experiences. According to the sensory conflict theory, the motion sickness would be occurred by this kind of the divergence.

Physiological and psychological measurements are used to evaluate the influence of VIMS on the body. The latter method to evaluate VIMS is the simulator sickness questionnaire [8]. On the other hand, physiological evaluation of VIMS has been conducted using parameters such as the high- and low- frequency components of electrogastrography, skin resistance, blood pressure, heart rate volubility, sweat rate, number of blinks, and respiratory rate [9$11]$.

A unique rhythm is associated with each variation of a physiological phenomenon. These include short repeated cycles, such as pulses, which are repeated in units of seconds, and long cycles, such as seasonal variations, which repeat in units of years. For instance, we can observe the circadian-dependent variability in the human blood pressure [12]. This is known as intra-day blood pressure fluctuation and can have an intra-day variation pattern characterized by high blood pressure in the daytime and decreased blood pressure at night [13]. Autonomous nerve activity 


\section{Y. Matsuura et al. / Advances in Science, Technology and Engineering Systems Journal Vol. 4, No. 4, 42-46 (2019)}

considerably affects this variability. Blood pressure increases or decreases depending on the variations in circulating blood volume and postural blood pressure reflex activity due to variations in posture. Heartrate and blood pressure intra-day variations are known to act as an evaluation index for autonomic nervous function. Additionally, the head-up tilt test is known as an autonomic nervous functional test associated with passive postural change [14]. Heart rate in healthy individuals increases, and the R -R interval estimated from the Electrocardiogram (ECG) decreases when using a tilt-table to switch from a supine to standing position [14]. Also, the same effects are reportedly observed when switching from a supine to seated position [15]. In these cases, the blood is accumulated in the lower extremities, however, vasoconstriction is automatically conducted to prevent from the hypostasis. The arterial baroreceptor is known as an sensory organ to detect the variations in blood pressure owing to the postural changes and other activities. This bio-signal reaches the brain stem vasomotor center (BSVC) through afferent pathways in the medium of vagus or glossopharyngeal nerve. The BSVC is activated with decline in the activity of the afferent pathways when the blood pressure decreases, and an effect is observed in excitability toward the sympathetic nerve; Noradrenaline is secreted from vascular wall sympathetic nerve endings, and adrenaline is, therefore, secreted from the adrenal medulla. Hence, the blood pressure can be maintained. As venous return decreases, the vagus nerve activity in the afferent pathway decreases through the low-pressure receptors in pulmonary veins and the atrium. As this information is received by the brain stem and reaches the hypothalamus, arginine vasopressin is secreted from the posterior pituitary, and an increased effect occurs in vasoconstriction and circulating plasma volume. Although cardiocirculatory research after postural changes is readily available, studies regarding the impact of posture changes on electrogastrograms are limited.

Percutaneous electrogastrography allows to noninvasively examine gastric myoelectrical activity. Human gastric myoelectrical activity cannot be measured by any other conventional method such as computed tomography, gastrofiberscopes or magnetic resonance imaging. An electrogastrogram (EGG) is usually evaluated using Fourier analysis, running spectral analysis, and other spectrum analysis. However, the information retrieved by this analysis is limited, and EGGs are not as usual as electrocardiograms, electroencephalograms, and other biosignal measurements, thus compromising their development.

In 1921, Walter Alvarez [16] recorded the first human EGG. EGGs are recorded by placing $\mathrm{Ag}-\mathrm{AgCl}$ electrodes on the surface of the epigastrium. EGG records the electrical signals which travel through the stomach muscles and control the muscle contractions. Human gastric slow wave or pacesetter potential activity generated by the interstitial cells of Cajal (ICCs) occurs at a frequency of 3 cycles per minute [17] [18], as shown in Figure 1. The ICCs are known as pacemaker cells that spontaneously depolarize and repolarize. The ICCs set the myoelectrical rhythmicity of the stomach and other areas of gastrointestinal tract. Recorded EGG signals that have noise with higher frequencies $>0.15 \mathrm{~Hz}$ must be filtered out [19]. These filters such as a low-pass filter, a band-pass filter eliminate most respiratory and cardiac rhythms in electromyogram.

Many previous studies on electrogastrography have been conducted but have been mostly related to the clinical field [20]. For instance, hormone, drugs, and motion sickness effects on EGG have been evaluated. Likewise, EGG has been used to study the

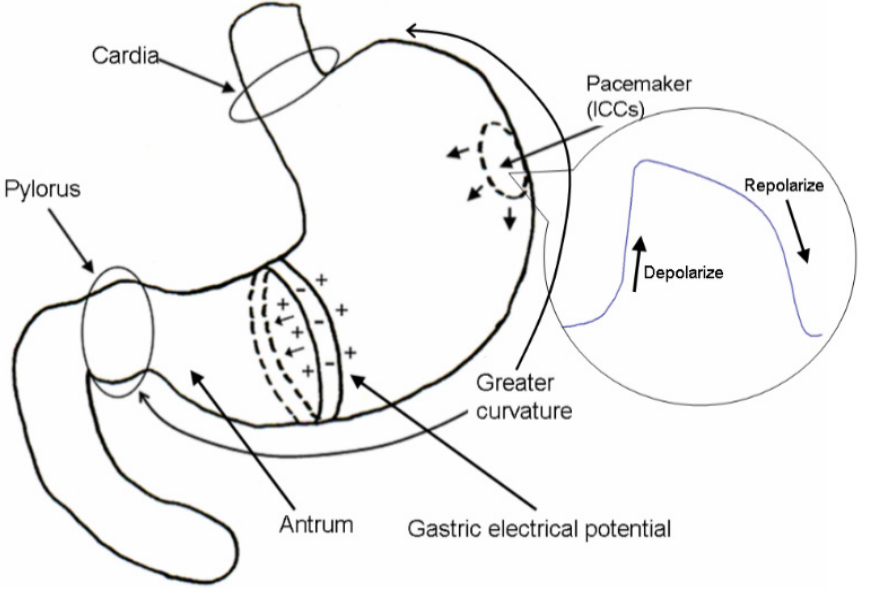

Figure 1: Traveling slow waves. The ICCs set the myoelectrical rhythmicity of the stomach and other areas of gastrointestinal tract.

effects of warm compresses on both gastrointestinal activity [21] and the epigastric region for relieving constipation [22], and to characterize the intestinal activity in patients with chronic constipation [23]. However, studies on clinical applications mainly consider patients, and hence scarce research is available on the EGG dynamics of healthy subjects.

More specifically, few studies have been focused on gastrointestinal motility in seated and supine positions to evaluate the severity of motion sickness. As it has been reported that threedimensional motion sickness is strongly elicited when viewing stereoscopic movies for prolonged periods, it is important to assess the safety while viewing such movies. Therefore, in this study the authors compared EGGs in seated and supine positions using the Wayland algorithm to evaluate motion sickness induced by stereoscopic movies.

\section{Material and Method}

The participants were 5 healthy females aged 19 to 24 years. The Ethical Committee of Graduate School of Natural Sciences, Nagoya City University approved the experiment. Prior to the experiment, each participant signed their informed consents.

The design of experiment is Figure 2. This experiments was conducted, considering the order effect in the protocol. In Addition, postural change on experiment $\mathrm{B}$ is passive position change to remove the effect of muscle sympathetic nerve activity.

The experimental room and chair condition is Table 1 . The experimental condition is Table 2. Participants finished their meals 120 mins beforehand. Therefore, the experiments were not affected by the presence of food.

Electrodes of EGG pasted on abdominal surface using 2 disposable $\mathrm{Ag}-\mathrm{AgCl}$ electrodes as shown in Figure 3. Their abdominal surface of the pasting points was cleaned out with cotton moistened with ethanol and the skin abrasion (SkinPure, Nihon Kohden, Japan).

Using A/D converter, the analog signals of their EGGs were A/D-converted to digital signals at $1 \mathrm{kHz}$. A low-pass filter of $0.15 \mathrm{~Hz}$ eliminated the activity in respiratory, cardiac rhythms, electromyogram, and the noise of electronic devices. In addition, resampling was performed every $1 \mathrm{~Hz}$ to remove the noise in the abovementioned measurement. 


\section{Experiment A (Seated)}

\begin{tabular}{|c|c|}
\hline $\begin{array}{c}\text { Seated Posture } \\
\text { (Rest) } \\
30 \mathrm{~min}\end{array}$ & Seated Posture \\
$\mathrm{t}_{0}$ & $90 \mathrm{~min}$ \\
\hline
\end{tabular}

\section{Experiment B (Supine)}

\begin{tabular}{|c|c|}
\hline $\begin{array}{c}\text { Seated Posture } \\
\text { (Rest) }\end{array}$ & Supine Posture \\
$30 \mathrm{~min}$ & $90 \mathrm{~min}$ \\
\hline
\end{tabular}

$t_{0}$

Figure 2: Experiments A and B

Table 1: Experimental room and chair condition

\begin{tabular}{|l|l|}
\hline \multicolumn{1}{|c|}{ Items } & \multicolumn{1}{c|}{ Conditions } \\
\hline Room Condition & Without windows \\
\hline Sound-insulated & $40 \mathrm{~dB}$ \\
\hline Room temperature & $20-24^{\circ} \mathrm{C}$ \\
\hline Air current & below $0.1 \mathrm{~m} / \mathrm{s}$ \\
\hline $\begin{array}{l}\text { Chair size } \\
\text { (Seating surface) }\end{array}$ & $55 \mathrm{~cm}($ width $) \times 55 \mathrm{~cm}($ Length) \\
\hline $\begin{array}{l}\text { Chair size } \\
\text { (Seating back) }\end{array}$ & $55 \mathrm{~cm}($ width) $\times 58 \mathrm{~cm}($ Length) \\
\hline $\begin{array}{l}\text { Chair angle between } \\
\text { the seating surface } \\
\text { and the back }\end{array}$ & 100 degrees \\
\hline
\end{tabular}

Table 2 Experimental condition and machines

\begin{tabular}{|l|l|}
\hline \multicolumn{1}{|c|}{ Items } & \multicolumn{1}{c|}{ Condition/Machine } \\
\hline Measurement Start time & $14: 00-15: 00$ \\
\hline EGGs Sampling Rate & $1 \mathrm{KHz}$ \\
\hline Bio-amplifier & MT11; NEC Medical, Japan \\
\hline Recorder & $\begin{array}{l}\text { PC216Ax; Sony Precision } \\
\text { Technology, Japan }\end{array}$ \\
\hline A/D converter & $\begin{array}{l}\text { AD16-16U (PCI) EH; CONTEC, } \\
\text { Japan }\end{array}$ \\
\hline Electrodes & Vitrode Bs, Nihon Kohden, Japan \\
\hline
\end{tabular}

Every 300-second, we analyzed time series data of the EGG for a 1024-second time window to divide the data (Figure 4). In this study, the authors calculated the translation error $\left(\mathrm{E}_{\text {trans }}\right)$ using Wayland algorithm from EGG time series data in 1 to 10 dimensional phase space [24-26].

\section{Results}

EGGs in seated (experiment A) and supine (experiment B) positions of healthy women over $5 \mathrm{~min}$ after $70 \mathrm{~min}$ from the beginning of measurements are shown in Figures 5(a) and (b), respectively. The EGG in seated position (Figure 5(a)) shows a large amplitude and unstable fluctuations. In contrast, the EGG in supine position (Figure 5(b)) shows a regular pattern. Figures 6(a) and (b) show the two-dimensional attractors $(\tau=3)$ generated from the EGGs in seated (Figure 5(a)) and supine (Figure 5(b)) positions, respectively.

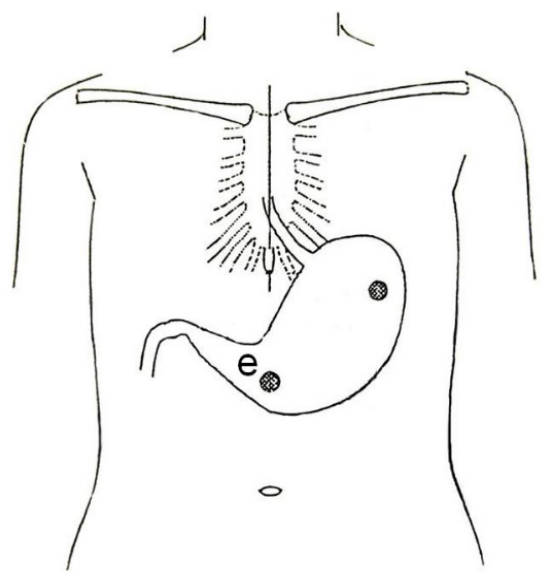

Figure 3: Electrodes position pasted on the surfece of body.

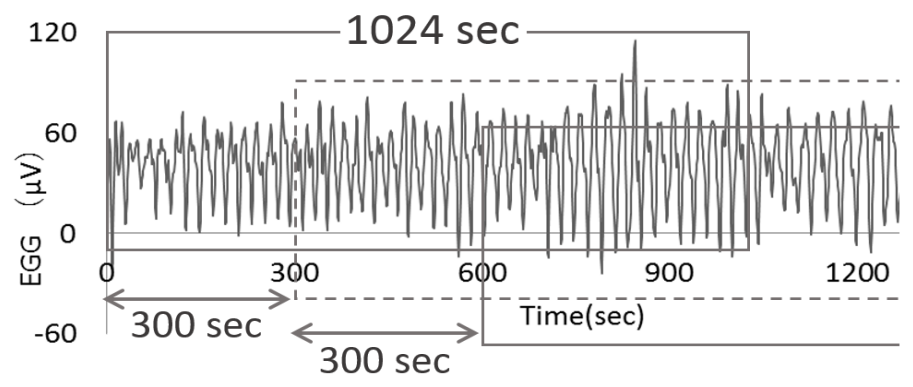

Figure 4: Moving segment of EGG-data.

(a)

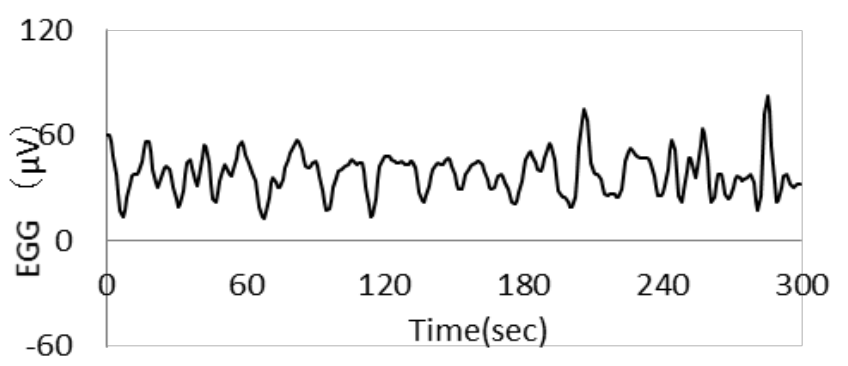

(b)

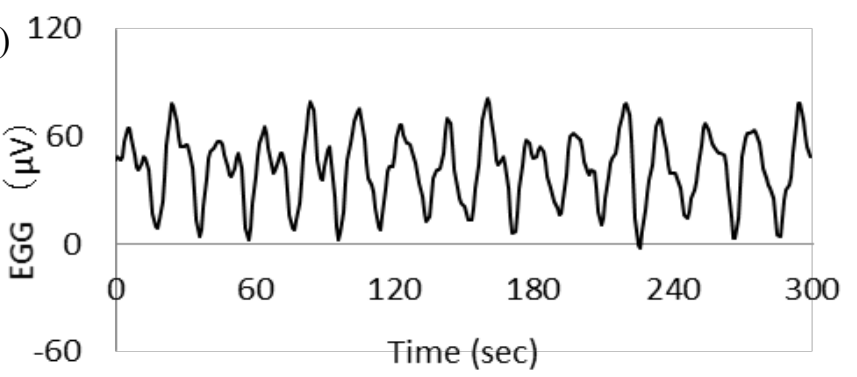

Figure 5: Typical examples of EGG in (a) experiment A and in (b) experiment B.

The EGG translation errors over a ten-dimensional embedding space in the seated position (excluding control) ranged from 0.27 to 0.58 , with average of 0.46 and standard deviation of 0.07 . The similar EGG translation errors in the supine position ranged from 0.23 to 0.52 , with average of 0.40 and standard deviation of 0.08 . The authors compared the translation errors estimated from the EGGs of experiment A with those of experiment B. Variations of $z$-scores of translation error (average \pm standard error) were 
estimated from the EGGs of the individuals in the ten-dimensional embedding space (Figure 7). The $z$-scores in the seated position (excluding control) ranged from -2.48 to 2.45 , with average of 0.07 and standard error of 0.42 , and those in the supine position ranged from -1.63 to 3.18 , with average of 0.04 and standard error of 0.42 .

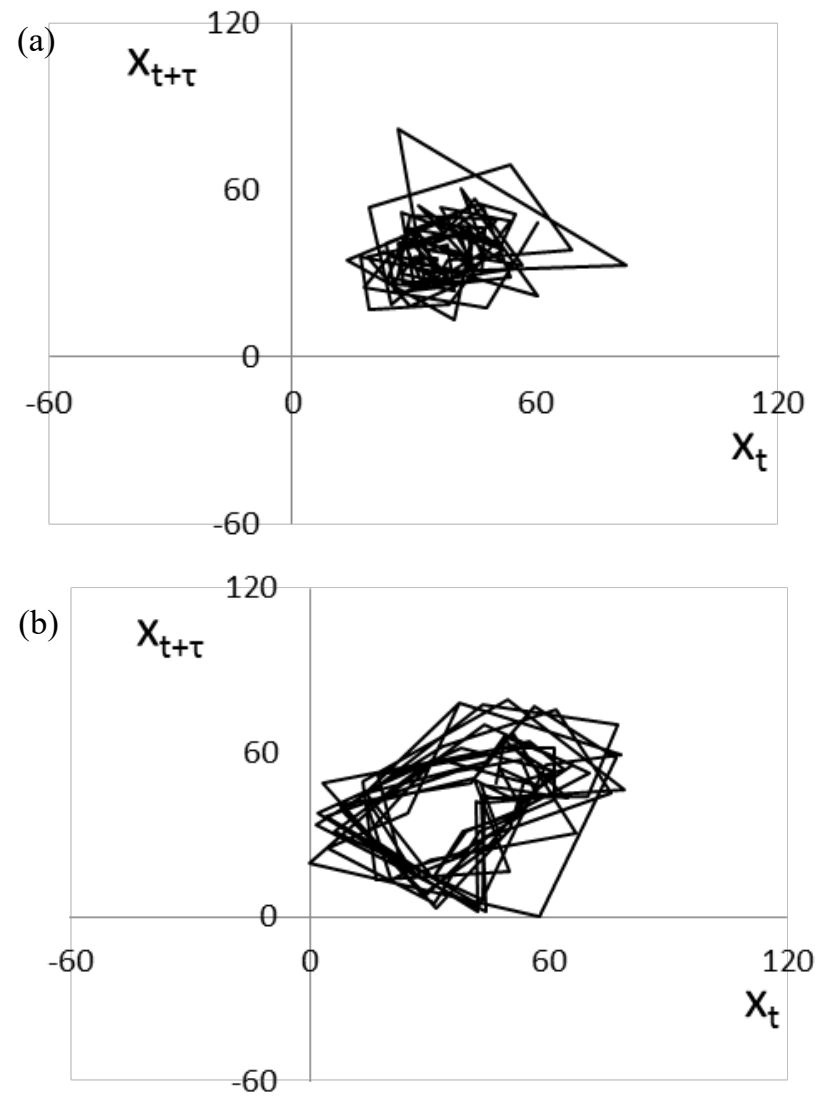

Figure 6: Attractors of EGG in (a) experiment A (Figure 5(a)) and in (b) experiment B (Figure 5(b)).

Then, the values of the translation errors estimated from the EGGs of experiments A and B were compared using the Wilcoxon signed-rank test. Significant differences were observed 50 and 55 min after the end of the resting period with $p<0.05$. The authors confirmed that translation errors in the ten-dimensional embedding space are sufficiently stable compared to those in embedding spaces with lower dimension. Figure 8 shows variations of the difference between the translation error estimated from the EGG at time $t$ and that at onset time $t_{0}$, except for the resting period in the ten-dimensional embedding space. The variations were calculated as DTE in the seated position (excluding control) ranged from -0.19 to 0.13 , with average of -0.001 and standard error of 0.035 , and that in the supine position ranged from -0.13 to 0.086 , with average of -0.011 and standard error of 0.021 .

\section{Discussion}

To estimate the EGG dynamics, the authors analyzed EGG signals using the Wayland algorithm. The regularity and periodicity of the EGGs was considered the cause of the abovementioned results. As shown in Figures 7 and 8, significant differences in translation errors estimated from EGGs in seated and supine positions were determined after 50 and 55 min from the resting period.

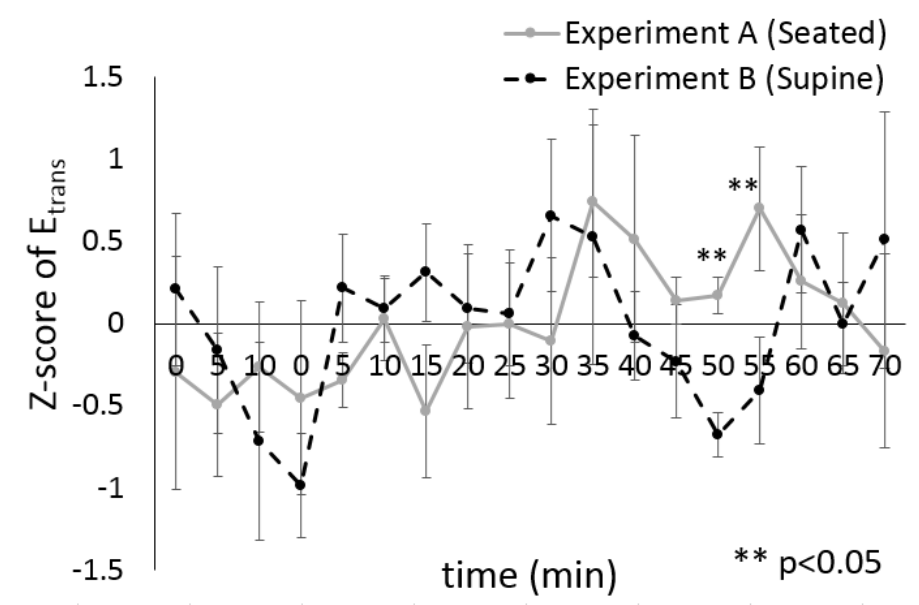

Figure 7: Z-scores of translation error (average \pm standard error).

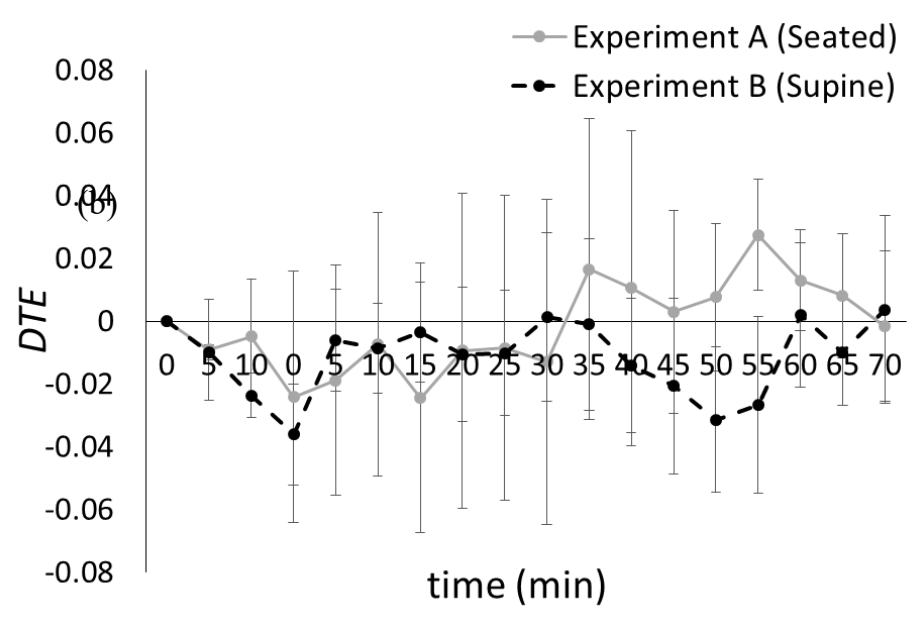

Figure 8: Difference translation errors (average \pm standard error).

$$
D T E(t)=E_{\text {trans }}(t)-E_{\text {trans }}\left(t_{0}\right) .
$$

Some studies have reported about the effect of postural change on the cardiocirculatory system after subjects changes from a supine to seated/standing postures, however, few does not have studied the effect of postural change on the autonomic nervous system, especially on the EGGs recorded over a prolonged period. Measuring bowel sounds, Kobayashi et al. tried to investigate the effect of the postural changes on the intestinal peristalsis [27]. Healthy middle-aged women voluntary participated in this study. Their bowel sounds were recorded during the right lateral recumbent following the supine for an hour. The results showed that intestinal peristalsis was significantly rose 30-75 min after the postural change whereas these results are questionable owing to the considerable differences prevalent among the participants [27]. In the other previous work, it has been reported that intestinal peristalsis would be temporarily suppressed if the intra-abdominal organ were affected by sudden postural changes [28]. Recording the bowel sounds $30 \mathrm{~min}$ after the postural change to the supine is appropriate for stationary measurements [29]. These results suggest that the stomach or intestines become active by means of parasympathetic nerve activity acceleration after a determined amount of time has passed since resuming supine position.

In general, EGGs are recorded in either a seated or supine position [30], and motion sickness is more often induced in the seated than in the supine position. In addition, the effect of postural 
change on the autonomic nervous system, especially in the digestive system, has been investigated in some studies, although with focus on the cardiovascular system [31][32]. In this study, the authors conducted a basic experiment to investigate the effect of postural change on the autonomic nervous system with focus on the digestive system.

\section{Conclusions}

Motion sickness and diseases are usually settled in a seated position. In fact, humans can hardly get motion sickness in a supine position. This study aimed to compare EGGs acquired in seated and supine positions using the Wayland algorithm to evaluate motion sickness induced by stereoscopic movies. The authors verified that the supine position retrieves more stable EGG signals in the supine than in the seated position, thus confirming the benefits of the supine position to relieve motion sickness. There are various cycles that occur in biorhythms, and many of the variations are affected by regional differentiation of the sympathetic system. Biorhythms are also impacted by variations in posture as well as the physical condition of a person and their lifestyle. Integrative and multifaceted evaluation of impact from biorhythm and posture variations is therefore useful for evaluating autonomic nervous function and estimating biological condition. The lack of research on gastric motility and intestinal peristalsis due to variation in posture warrants an ongoing necessity for accumulation of foundational research. In upcoming studies, the authors will measure heart rate, blood pressure and multichannel EGG signals in seated and supine positions.

\section{Conflict of Interest}

The authors declare no conflict of interest.

\section{Acknowledgment}

This study was supported in part by the Kayamori Foundation of Informational Science Advancement, the Japan Society for the Promotion of Science, and Grant-in-Aid for Scientific Research (C) (Grant numbers 17K00715 and 18K11417).

\section{References}

[1] G.W. Manning, G.W. Stewart, "Effect of body position on incidence of motion sickness," J Appl Physiol. 1(9), 619-628, 1949.

[2] J.T. Reason, J.J. Brand, Motion Sickness, 3rd ed, San Diego: Academic Press Inc, 1975.

[3] J. A. Irwin, "The pathology of sea-sickness," The Lancet. 118(3039), 907909, 1878.

[4] B. Keshavarz, H. Hecht, B. D. Lawson., "Visually induced motion sickness: Causes, characteristics, and countermeasures," in Handbook of Virtual Environments, 2nd Edition, K. S. Hale, K. M. Stanney, Ed, FL, CRC Press, 2015, 647-698.

[5] N.H. Barmack, "Central Vestibular System: Vestibular nuclei and posterior cerebellum,” Brain Res Bull 60(5-6), 511-541, 2003.

[6] C.D. Balaban, J.D. Porter, "Neuroanatomical substrates for vestibuloautonomic interactions," J Vestib Res. 8, 7-16, 1998.

[7] N. Takeda, M. Morita, T. Kubo, A. Yamatodani, T. Watanabe, H. Wada, T. Matsunaga, "Histaminergic mechanism of motion sickness neurochemical and neuropharmacological studies in rats," Acta Otolaryngol. 101(5-6), 416421, 1986.

[8] R.S. Kennedy, N.E. Lane, K.S. Berbaum, M.G. Lilienthal, "Simulator Sickness Questionnaire: An Enhanced Method for Quantifying Simulator Sickness," Int J Aviat Psychol. 3(3), 203-220, 1993.

[9] S.R. Holmes, M.J. Griffin, "Correlation Between Heart Rate and the Severity of Motion Sickness Caused by Optokinetic Stimulation," J Psychophysiol, 15, 35-42, 2001.

[10] N. Himi, T. Koga, E. Nakamura, M. Kobashi, M. Yamane, K. Tsujioka, "Differences in autonomic responses between subjects with and without nausea while watching an irregularly oscillating video," Auton Neurosci. 116(1-2), 46-53, 2004.

[11] Y. Yokota, M. Aoki, K. Mizuta, Y. Ito, N. Isu, "Motion sickness susceptibility associated with visually induced postural instability and cardiac autonomic responses in healthy subjects," Acta Otolaryngol. 125(3), 280-285, 2005.

[12] R.C. Hermida, D.E. Ayala, J.R. Fernandex, L.M. Ruilope, J.E. Lopez, "Modeling the circadian variability of ambulatorily monitored blood pressure by multiple-component analysis", Chronol Int., 19, 461-481, 2002.

[13] A.M. Birkenhanger, A.H. van den Meiracker, "Causes and consequences of a non-dipping blood pressure profile”, Netherland J Med., 65, 127-131, 2007.

[14] S. Iwase, T. Mano, M. Saito, "Effects of graded head-up tilting on muscle sympathetic activities in man", Physiologist, 20, S62-S63, 1987.

[15] H. Noro, I. Watanabe, N. Yabunaka, Y. Ohtsuka, Y. Agishi, "Quantitative Evaluation of Changes in pulse Rate and Blood Pressure by the Supine - to Sitting active Postural Change Test", The Autonomic nervous system 32(2), 111-118, 1995.

[16] W.C. Alvarez, "The electrogastrogram and what it shows," JAMA. 78(15), 1116-1118, 1922.

[17] D. Couturier, C. Roze, J. Paolaggi, C. Debray, "Electrical activity of the normal human stomach: A comparative study of recordings obtained from the serosal and mucosal sides," Dig. Dis. Sci. 17(11) 969-976, 1972.

[18] E.J. Van Der Schee, A.J.P.M. Smout and J.L. Grashuis, "Application of running spectrum analysis to electrogastrographic signals recorded from dog and man," in Motility of the digestive tract, M. Wienbeck, Ed, New York: Raven Press, 1982, 241-250.

[19] J.Z. Chen, R.W. McCallum, Electrogastrography: Principles and Applications, New York: Raven Press, 1994.

[20] M. Nagai, M. Wada, Y. Kobayashi, S. Togawa, "Effects of lumbar skin warming on gastric motility and blood pressure in humans," Jpn. J. of Physiol. 53(1), 45-51, 2003.

[21] N. Kawachi, S. Iwase, H. Takada, D. Michigami, Y. Watanabe, N. Mae, "Effect of wet hot packs applied to the epigastrium on electrogastrogram in constipated young women," Autonomic Nervous System 39(5), 433-437, 2002.

[22] Y. Matsuura, S. Iwase, H. Takada, Y. Watanabe, E. Miyashita, "Effect of three days of consecutive hot wet pack application to the epigastrium on electrogastrography in constipated young women," Autonomic Nervous System. 40(4), 406-411, 2003.

[23] S.R. Cajal, Histologie du systeme nerveux de l'homme et des vertebras, Vol. 2. Paris :Maloine, 1909, p. 942. (in French); S.R. Cajal, N. Swanson, L.W. Swanson, Histology of the nervous system of man and vertebrates, New York: Oxford University Press, 1995. (reprinted in English)

[24] R. Wayland, "Recognizing Determinism in a Time Series," Phys. Rev. Lett. 70-5, 580-582, 1993.

[25] H. Takada, T. Morimoto, H. Tsunashima, T. Yamazaki, H. Hoshina, M. Miyao, "Applications of Double-Wayland Algorithm to Detect Anomalous Signals," Forma, 21(2), 159-167, 2006.

[26] Y. Matsuura, H. Takada, K. Yokoyama, K. Shimada, "Proposal for a New Diagram to Evaluate the Form of the Attractor Reconstructed from Electrogastrography", Forma, 23, 25-30, 2008.

[27] T. Kobayashi, T. Yazaki, Y. Hujihara, H. Ozawa, J. Nakahashi, "Analysis of Change in Bowel Sound and Movement by the Posture Change: Using an Actigraph Micromini Type Sound Sensor", Bulletin of Faculty of Nursing, Yamanashi Prefectural University 13, 37-46, 2011.

[28] K. Fukai, N. Sakamoto, N. Tanaka, "Effect of Oral Water Intake, Exercise and Hot Compresses on Bowel Sounds in Healthy Women", Kawasaki medical welfare journal 6(1), 99-106, 1996.

[29] N. Sakamoto, K. Fukai, N. Tanaka, "The Relationship Between Bowel Sounds and Constipation Assessment in Healthy Women", Kawasaki medical welfare journal 6(2), 341-346, 1996.

[30] K. L. Koch, R. M. Stern, Handbook of Electrogastrography, New York: Oxford University Press, 2004.

[31] K. Nishijo, "The Relation between the Penpheral Clrculatory Behaviour and the Changes of the Body Posrtron”, Bulletin of defectology, 2, 33-45, 1978.

[32] S. Mukai, J. Hayano, "Heart rate and blood pressure variabilities during graded head-up tilt", J. Appl. Physiol., 78(1), 212-216, 1995. 